\title{
Clinical and microbiological profile of babies born with risk of neonatal sepsis
}

\author{
BIN ZHOU, XIAO LIU, JIE-BIN WU, BAO JIN and YAN-YAN ZHANG \\ Department of Pediatrics, Xuzhou Central Hospital, The Affiliated Xuzhou Hospital of Medical College \\ of Southeast University, Xuzhou Clinical Medical College of Nanjing University of Chinese Medicine, \\ Xuzhou Clinical School of Xuzhou Medical College, Xuzhou, Jiangsu 221009, P.R. China
}

Received February 8, 2016; Accepted August 23, 2016

DOI: $10.3892 /$ etm.2016.3836

\begin{abstract}
The aim of the present study was to evaluate the effects of antibiotics on the condition of babies born with risk of neonatal sepsis. From March, 2014 to February, 2015, 200 neonates born with risk factors of septicemia in the Neonatal Intensive Care Unit at Xuzhou Central Hospital, were enrolled in the present study. Venous blood samples were collected within $6 \mathrm{~h}$ of birth using aseptic technique. Part of the blood specimens were cultured using BACTEC PEDS PLUS/F Culture Vials. Subsequently, the subcultures were prepared from each presumptive positive vial and bacterial isolates were identified. The remaining portion was used to measure the level of C-reactive protein (CRP) and total leukocyte count (TLC). The result showed that $32 \%$ of neonates were infected, of whom, 21.9\% had Staphylococcus aureus, 21.9\% had Acinetobacter Baumanni, and $12.5 \%$ had Klebsiella pneumoniae. Additionally, Staphylococcus epidermis, Enteroccus spp., Pseudomonas aeruginosa and $E$. coli was isolated from 9.4, 7.8, 6.3 and $4.7 \%$ of neonates, respectively. The neonates enrolled in the present study had $\geq 1$ risk factor for neonatal sepsis, and the average number of risk factors was 1.95 per neonate. Neonates $(39.1 \%)$ with positive blood culture results, had a CRP level $>0.8 \mathrm{mg} / \mathrm{dl}$, and $12.5 \%$ was shown to have an abnormal increase in their leukocyte counts. The association between leukocyte counts and blood culture results was not statistically significant. Of the neonates with positive blood cultures $45.3 \%$ died within 7 days after birth, while there was no mortality among those with negative culture results. The results indicate that in the presence of risk factors for sepsis, irrespective of clinical features of septicemia, neonatal sepsis screening should be performed. Rational and appropriate use of antibiotics may minimize the emergence of multidrug resistant bacteria in neonatal units.
\end{abstract}

Correspondence to: Dr Xiao Liu, Department of Pediatrics, Xuzhou Central Hospital, 199 Jiefang Road, Xuzhou, Jiangsu 221009, P.R. China

E-mail:1liuxiao420@163.com

Key words: neonatal sepsis, septicemia, C-reactive protein

\section{Introduction}

Neonatal sepsis is defined as a clinical syndrome of bacteremia with systemic signs and symptoms of infection in the first 4 weeks of life (1). Septicemia occurs in $2.3 \%$ of intramural live births (2). Over $40 \%$ of the under-5 deaths globally occur in the neonatal period (3). The World Health Organization estimates that $>1$ million neonatal deaths worldwide annually are caused by severe infections, and $\sim 1$ million deaths are due to neonatal sepsis or pneumonia alone (4). Morbidity of neonatal sepsis differs significantly from country to country. The incidence of neonatal sepsis varies from 1 to 5 cases per 1,000 live births in developed countries, but increases in developing countries, varying from 49 to 170 per 1,000 (5). Neonatal sepsis can be classified into two types based on the postnatal ages at onset: Early-onset neonatal sepsis (EOS) occurs in the first seven days, whereas late-onset neonatal sepsis (LOS) occurs after the seventh day. EOS is caused by microorganisms from the maternal genital tract before or at the time of birth, while LOS is due to organisms acquired after delivery and considered nosocomial community-acquired infections (6).

Early empirical antibiotic treatment of neonates suspected of having septicemia is the standard practice. Nonetheless, the dilemma of unnecessary exposure to antibiotics in this vulnerable population remains, creating an environment for emerging bacterial resistance and the potential for poor prognosis (7). On the other hand, the survivors of neonatal sepsis are vulnerable to short- and long-term neuro-developmental morbidity (8-10). Neonatal sepsis is caused by Gram-positive and Gram-negative bacteria and Candida (11). The diversity of organisms causing sepsis varies in different regions and changes over time even in the same location $(12,13)$. This is attributed to the changing pattern of antibiotic use and changes in lifestyle. Many factors contribute to the susceptibility of the neonate to sepsis, which can influence the incidence of neonatal sepsis (11). Incidence also varies from nursery to nursery depending on conditions predisposing infants to infection $(11,14)$.

\section{Materials and methods}

Study design and population. This was a prospective study conducted over a period of 12 months between March, 2014 
Table I. Maternal and neonatal data of enrolled study participants $(n=200)$.

\begin{tabular}{lr}
\hline Characteristics & No. $(\%)$ \\
\hline Maternal data & \\
Gestational age & $114(57.0)$ \\
Preterm $(<37$ weeks) & $86(43.0)$ \\
Term ( $\geq 37$ weeks $)$ & \\
Mode of delivery & $72(36.0)$ \\
Vaginal & $128(64.0)$ \\
Caesarian section & \\
Neonatal data & $54(27.0)$ \\
Birth weight & $84(42.0)$ \\
VLBW $(\leq 1,500 \mathrm{~g})$ & $62(31.0)$ \\
LBW $(1,501-2,500 \mathrm{~g})$ & \\
Normal $(>2,500 \mathrm{~g})$ & $90(45.0)$ \\
Gender & $110(55.0)$ \\
Male & \\
Female & $64(32.0)$ \\
Blood culture & $136(68.0)$ \\
Positive & \\
Negative & \\
\hline
\end{tabular}

VLBW, very low birth weight; LBW, low birth weight.

Table II. Risk factors of neonatal sepsis present in study participants $(\mathrm{n}=200)$.

\begin{tabular}{lc}
\hline Risk factors & No. of babies (\%) \\
\hline PROM & $8(4.0)$ \\
Amnionitis & $12(6.0)$ \\
Meconium-stained liquor & $58(29.0)$ \\
Low birth weight $(<2.5 \mathrm{~kg})$ & $138(69.0)$ \\
Preterm (<37 weeks) & $114(57.0)$ \\
$\geq 3$ per vaginal examinations during labour & $2(1.0)$ \\
Active resuscitation required in labour room & $12(6.0)$ \\
Dai handling & $6(3.0)$ \\
Fever in the mother during & $38(19.0)$ \\
Labour (temperature of $\left.\geq 38^{\circ} \mathrm{C}\right)$ & $2(1.0)$ \\
Urinary tract infection in the mother & \\
\hline
\end{tabular}

PROM, preterm premature rupture of membranes.

and February, 2015, at the Neonatal Intensive Care Unit in Xuzhou Central Hospital (Xuzhou, China). During the study period, neonates born with the risk of neonatal sepsis were enrolled for the study after obtaining informed consent from the parents. This study was approved by the Ethics Committee of Xuzhou Children's Hospital.

The inclusion criteria were: Babies were required to have $\geq 1$ of the following risk factors of sepsis mentioned
Table III. Blood culture positivity according to the number of risk factors present $(\mathrm{n}=200)$.

No. of risk No. of positive

factors present No. of neonates blood cultures (\%) P-value

\begin{tabular}{lrrr}
\hline 1 & 42 & $8(19.1)$ & 0.001 \\
2 & 124 & $34(27.4)$ & 0.001 \\
$\geq 3$ & 34 & $22(64.7)$ & 0.001 \\
\hline
\end{tabular}

below, premature rupture of membranes, Amnionitis, Meconium stained liquor, low birth weight (LBW) $(<2.5 \mathrm{~kg})$ 3 per vaginal examinations during labour, preterm infants, active resusitation required in the labor room, mother having temperature of $\geq 38^{\circ} \mathrm{C}$, urinary tract infection in the mother. Preterm $(<37$ weeks). The exclusion criteria were: Babies born to mothers who had received antenatal antibiotic therapy within $48 \mathrm{~h}$ prior to the delivery or had major congenital anomaly.

During the study period of 12 months, 200 consecutive neonates born with risk factors of septicemia were studied. Of these 90 were males and 110 were females. After recruitment 1.5-2 ml venous blood sample was collected within $6 \mathrm{~h}$ of birth using aseptic technique, 0.5-1 $\mathrm{ml}$ of which was directly transferred into the BACTEC PEDS PLUS/F Culture Vials (BD Biosciences, Franklin Lakes, NJ, USA). The remaining portion was used for estimation of C-reactive protein (CRP) and total leukocyte count (TLC). The samples were transported at room temperature as early as possible to microbiology laboratory for further processing.

The inoculated BACTEC vials were placed in a BACTEC 9120 system. A positive result was indicated by an audible alarm and yellow illumination of the positive indicator lamp at the site of positive vial. On the computer instrument status display the station number was shown by flashing green in case of a positive vial. The bottles were incubated for 5 days before being reported as negative. A gram stain and a subculture on blood agar and MacConkey agar were performed from each presumptive positive vial. After incubation at $37^{\circ} \mathrm{C}$ the bacterial isolates were identified by gram staining, colony characteristics and a battery of biochemical tests, following a standard protocol (15). TLC was done by automated analyser-Coulter LH 500. A total of 5,000-25,000 leukocytes/ $\mu 1$ was taken as normal. CRP latex slide test (SmarTest Diagnostics, London, UK) was used for the semi-quantitative measurement of CRP in human serum. The threshold value was taken as $0.8 \mathrm{mg} / \mathrm{dl}$.

Statistical analysis. Data were collected by using a questionnaire, master chart was prepared in excel and statistical analysis was performed in line with objectives. Continuous variables are presented as mean \pm standard deviation and categorical variables as counts or percentages. Correlation of the risk factors with laboratory findings was obtained by using the Pearson Chi-square test and Fisher's exact test. $\mathrm{P}<0.05$ was considered to indicate a statistically significant difference. 
Table IV. Correlation of CRP and TLC with blood culture positivity.

\begin{tabular}{|c|c|c|c|c|c|c|}
\hline \multirow[b]{3}{*}{ Characteristics } & \multicolumn{4}{|c|}{$\mathrm{CRP}$ in $\mathrm{mg} / \mathrm{dl}$} & \multicolumn{2}{|c|}{ TLC per $\mu 1$} \\
\hline & \multirow{2}{*}{$\frac{\text { Normal }}{0.8}$} & \multicolumn{3}{|c|}{ Abnormal } & \multirow{2}{*}{$\frac{\text { Normal }}{5,000-25,000}$} & \multirow{2}{*}{$\frac{\text { Abnormal }}{>25,000}$} \\
\hline & & $0.8-1.5$ & $1.6-3.1$ & $\geq 3.2$ & & \\
\hline \multicolumn{7}{|l|}{ Blood culture } \\
\hline Positive $(n=64)$ & 39 & 2 & 11 & 12 & 56 & 8 \\
\hline Negative $(n=136)$ & 108 & 28 & - & - & 136 & - \\
\hline P-value & 0.015 & 0.012 & & & 0.064 & \\
\hline
\end{tabular}

CRP, C-reactive protein; TLC, total leukocyte count.

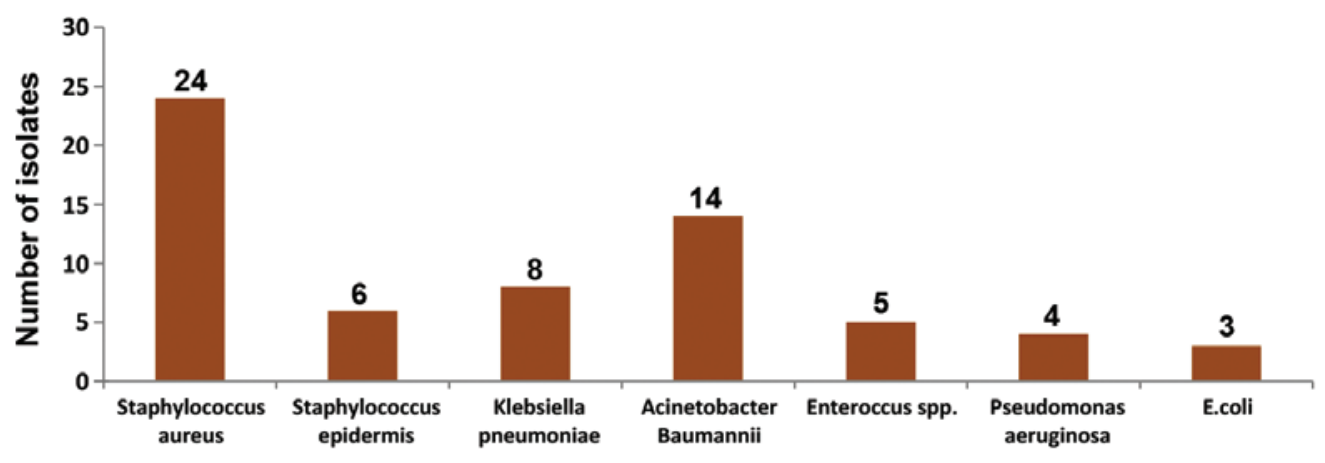

Figure 1. Profile of bacterial isolates from blood samples ( $n=64)$.

\section{Results}

Patient characteristics. Out of the total 200 newborn babies enrolled for the study, $110(55 \%)$ were females and $90(45 \%)$ were males; 128 (64\%) of the babies were delivered through caesarian section and $72(36 \%)$ vaginally; $114(57 \%)$ of the babies were preterm; $62(31 \%)$ of the babies were of normal weight, 84 (42\%) were of LBW and 54 (27\%) of the babies were of very LBW. Of the babies enrolled and subjected for blood culture, 64 (32\%) were positive and 136 (68\%) were negative on blood culture (Table I). Of the 64 culture-positive cases, 24 (37.5\%) were Staphylococcus aureus, 14 (21.9\%) were Acinetobacter Baumanni, 8 (12.5\%) were Klebsiella pneumoniae, 6 (9.4\%) were Staphylococcus epidermis, 5 (7.8\%) were Enteroccus spp., 4 (6.3\%) were Pseudomonas aeruginosa and 3 (4.7\%) were E. coli (Fig. 1).

Risk factors. Of the total 200 babies enrolled for the study all has 1 or more than 1 risk factors for neonatal sepsis. The average number of risk factors was 1.95 per child and maximum number of risk factors present in a child was 4 . Of the babies 138 (69\%) were of LBW followed by preterm $(57 \%)$ and meconium stained liquor (29\%). Of the mothers 38 (19\%) had fever during labour, 6\% had Amnionitis and required resuscitation. $8(4 \%)$ had preterm premature rupture of membranes, $6(3 \%)$ had history of indigenous method of delivery $1 \%$ had UTI in mother and required $\geq 3$ per vaginal examinations during labour (Table II).
Of the 200 cases 124 (62\%) had a minimum of 2 risk factors, $42(21 \%)$ had a minimum of 1 risk factor and $34(17 \%)$ had $\geq 3$ than 3 risk factors present. The blood culture positivity increased with rising number of risk factors. It was 64.7, 27.4 and 19.1\% with $\geq 3,2$ and 1 risk factors, respectively (Table III).

Blood culture. CRP and TLC values of the blood culture-positive and -negative samples are shown in Table IV. Of the 64 positive blood cultures, 25 (39.1\%) had CRP $>0.8 \mathrm{mg} / \mathrm{dl}$, whereas among the 136 blood culture-negative neonates, $28(20.6 \%)$ had CRP $>0.8 \mathrm{mg} / \mathrm{dl}$. The association between CRP and blood culture was statistically significant $(\mathrm{P}=0.015)$. Abnormal increased leukocyte counts were seen in $8(12.5 \%)$ of 64 positive blood cultures, whereas in all the blood culture-negative neonates, leukocyte count was normal. The association between leukocyte count and blood culture results was not statistically significant $(\mathrm{P}=0.064)$.

Of the 64 culture-positive neonates, 29 (45.3\%) succumbed within seven days, of whom 18 (62\%) were males and $11(38 \%)$ were females. There was no mortality among the culture negative neonates. The mean birth weight of the neonates who died was $1,468 \pm 468 \mathrm{~g}$, and their mean gestational age was $31.3 \pm 0.52$ weeks.

\section{Discussion}

The bacteriological profile of neonatal sepsis varies from location to location. In the United States, Group B Streptococcus 
is the leading cause of neonatal septicemia (16). However, according to the recent (2002-2003) national neonatal perinatal database in China, Klebsiella pneumoniae are the most common bacteria causing neonatal septicemia, followed by Staphylococcus aureus (2). In the present study Staphylococcus aureus, is the commonest microorganism isolated from the blood culture positive cases. Out of 64 positive cases 24 (37.5\%) were Staphylococcus aureus. The difference may be due to variations in population characteristics and in predisposing factors. These findings are corroborated by two more studies on neonatal septicemia conducted by Muhammad et al (17) and Agnihotri et al (18), respectively. In the two studies Staphylococcus aureus was the most common isolate being present in $27 \%$ cases in the former and $35 \%$ in the latter.

Of the gram-negative bacteria obtained in the present study, Acinetobacter baumannii was the most common isolate. Acinectobacter species are gaining importance as a potential pathogen in neonatal septicemia because of frequent isolation in the recent years (19). In the present study, Acinetobacter baumannii was isolated in $21.9 \%$ of the total septicemia cases, which is in line with the incidence of Acinetobacter sepsis reported from China (6.5-31.5\%) (19). Acinetobacter septicemia is common in babies with intravenous catheterization and artificial ventilation $(19,20)$. The most important neonatal factor predisposing to infection is prematurity and/or $\operatorname{LBW}(19,21)$, since they often require prolonged intravenous access, endotracheal intubation or other invasive procedures that provide a portal of entry for infection. A study from Pakistan reported that prematurity and LBW are associated with increasing risk of Acinetobacter infection in neonates (22). Additionally, the use of the central venous catheter and mechanical ventilation were identified as significant risk factors for neonatal Acinetobacter baumannii septicemia in a Brazilian study (23).

Blood culture positivity in the present study was associated with the number of risk factors present. In the presence of a single risk factor, blood culture positivity was present in $19.1 \%$ cases. In the presence of two risk factors, blood culture positivity increased to $27.4 \%$, while in the presence of $\geq 3$ risk factors, blood culture positivity increased by $>3$ times to $64.7 \%$. It may be concluded that as the number of risk factors increase, risk of septicemia in the neonate also increases. Thus, screening tests for septicemia must be perform routinely in such cases.

In the present study, a significant association was found between CRP and positive blood culture results $(\mathrm{P}=0.015)$. Similar results were obtained in a study by Caldas et al (24) where elevated CRP levels were associated with culture-positive cases $(\mathrm{P}<0.05)$. This indicates the importance of CRP as a significant marker of septicemia. No significant association was found between leukocyte counts and neonatal septicemia in this study. Similar result was obtained in a study done in Philippines by Mayuga et al (25). In a study by Ottolini et al (26) it was found that TLCs are of limited value in the diagnosis of septicemia in newborns. TLCs are particularly unreliable indicator of infection during the first several hours of early-onset (within $48 \mathrm{~h}$ of birth) sepsis because these values are initially normal.

In conclusion, blood culture is the gold standard to diagnose the neonatal sepsis. Blood culture is significantly correlated with the risk factors of neonatal sepsis. In the presence of risk factors for sepsis, irrespective of clinical features of septicemia, neonatal sepsis screening should be done. Rational and appropriate use of antibiotics minimizes the emergence of multidrug resistant bacteria in neonatal units.

\section{References}

1. Paolucci M, Landini MP and Sambri V: How can the microbiologist help in diagnosing neonatal sepsis? Int J Pediatr 2012: 120139, 2012.

2. NNPD Network: National Neonatal-Perinatal Database - Report 2002-2003. National Neonatology Forum NNPD Network, New Delhi, India.

3. UNICEF, WHO, The World Bank, and The United Nations: Levels and Trends in Child Mortality - Report 2011. UNICEF, New York, NY, 2011.

4. Qazi SA and Stoll BJ: Neonatal sepsis: a major global public health challenge. Pediatr Infect Dis J 28 (Suppl 1): S1-S2, 2009.

5. Thaver D and Zaidi AK: Burden of neonatal infections in developing countries: A review of evidence from community-based studies. Pediatr Infect Dis J 28 (Suppl 1): S3-S9, 2009.

6. Al-Taiar A, Hammoud MS, Cuiqing L, Lee JK, Lui KM, Nakwan $\mathrm{N}$ and Isaacs D: Neonatal infections in China, Malaysia, Hong Kong and Thailand. Arch Dis Child Fetal Neonatal Ed 98: F249-F255, 2013.

7. Clark RH, Bloom BT, Spitzer AR and Gerstmann DR: Empiric use of ampicillin and cefotaxime, compared with ampicillin and gentamicin, for neonates at risk for sepsis is associated with an increased risk of neonatal death. Pediatrics 117: 67-74, 2006.

8. Stoll BJ, Hansen N, Fanaroff AA, Wright LL, Carlo WA, Ehrenkranz RA, Lemons JA, Donovan EF, Stark AR, Tyson JE, et al: Changes in pathogens causing early-onset sepsis in very-low-birth-weight infants. N Engl J Med 347: 240-247, 2002.

9. Ferreira RC, Mello RR and Silva KS: Neonatal sepsis as a risk factor for neurodevelopmental changes in preterm infants with very low birth weight. J Pediatr (Rio J) 90: 293-299, 2014.

10. Dammann O, Kuban KCK and Leviton A: Perinatal infection, fetal inflammatory response, white matter damage, and cognitive limitations in children born preterm. Ment Retard Dev Disabil Res Rev 8: 46-50, 2002.

11. Jumah DS and Hassan ML: Predictor of mortality outcome in neonatal sepsis. Medical Journal of Basrah University 25: 11-18, 2007.

12. Shrestha S, Adhikari N, Rai BK and Shreepaili A: Antibiotic resistance pattern of bacterial isolates in neonatal care unit. JNMA J Nepal Med Assoc 50: 277-281, 2010.

13. Ghotaslou R, Ghorashi Z and Nahaei MR: Klebsiella pneumoniae in neonatal sepsis: A 3-year-study in the pediatric hospital of Tabriz, Iran. Jpn J Infect Dis 60: 126-128, 2007.

14. Klein JO and Remington JS (eds): Current concepts of infection of the fetus and newborn infant. In: Infectious diseases of the fetus and newborn. WB Saunders, Philadelphia, PA, pp1-24, 2000.

15. Collee JG, Fraser AG, Marmion BP, Simmons A (eds): Bacteria and Related Organisms: In: Mackie and McCartney Practical Medical Microbiology. Elsevier India, Gurgaon, India, pp151-423, 2008.

16. Puopolo KM: Bacterial and fungal infections. In: Manual of neonatal care. Cloherty JP, Eichenwald EC and Stark AR (eds). 6th edition. Lippincott William and Wilkins, Philadelphia, PA, pp274-300, 2008.

17. Muhammad Z, Ahmed A, Hayat U, Wazir MS, Rafiyatullah and Waqas H: Neonatal sepsis: Causative bacteria and their resistance to antibiotics. J Ayub Med Coll Abbottabad 22: 33-36, 2010.

18. Agnihotri N, Kaistha N and Gupta V: Antimicrobial susceptibility of isolates from neonatal septicemia. Jpn J Infect Dis 57: 273-275, 2004.

19. De AS, Rathi MR and Mathur MM: Mortality audit of neonatal sepsis secondary to acinetobacter. J Glob Infect Dis 5: 3-7, 2013.

20. Sriram R: Correlation of blood culture results with the sepsis score and the sepsis screen in the diagnosis of neonatal septicemia. Int J Biol Med Res 2: 360-368, 2011. 
21. Stoll BJ, Behrman RE, Kliegman RM and Jenson HB: Infections of the neonatal infant: Pathogenesis and epidemiology. In: Nelson textbook of Pediatrics. 17th edition. Saunders and Imprint of Elsevier, Philadelphia, PA, pp627-632, 2004.

22. Saleem AF, Ahmed I, Mir F, Ali SR and Zaidi AK: Pan-resistant Acinetobacter infection in neonates in Karachi, Pakistan. J Infect Dev Ctries 4: 30-37, 2009.

23. von Dolinger de Brito D, Oliveira EJ, Abdallah VO, da Costa Darini AL and Filho PP: An outbreak of Acinetobacter baumannii septicemia in a neonatal intensive care unit of a university hospital in Brazil. Braz J Infect Dis 9: 301-309, 2005.
24. Caldas JPS, Marba STM, Blotta MHSL, Calil R, Morais SS and Oliveira RTD: Accuracy of white blood cell count, C-reactive protein, interleukin-6 and tumor necrosis factor alpha for diagnosing late neonatal sepsis. J Pediatr (Rio J) 84: 536-542, 2008.

25. Mayuga WAB and Isleta PFD: Clinical correlation of neonatal and maternal hematological parameters as predictors of neonatal sepsis. PIDSP J 9: 36-43, 2005.

26. Ottolini MC, Lundgren K, Mirkinson LJ, Cason S and Ottolini MG: Utility of complete blood count and blood culture screening to diagnose neonatal sepsis in the asymptomatic at risk newborn. Pediatr Infect Dis J 22: 430-434, 2003. 